\title{
The untapped impact: Struggles and subsequent condition of Metro Manila taxi industry amid operations of Transport Network Companies
}

\author{
$N J$ Beltran $^{1, *}, R$ De Jesus ${ }^{2}, L R$ Alipio $^{1}, E$ Maynite $^{1}, C$ Clemente $^{1}, \tilde{N} R$ Gonzales ${ }^{1}$, and $N J$ \\ Silagan $^{1}$ \\ ${ }^{1}$ Student, Senior High School Department, Dr. Yanga's Colleges Inc., Wakas, Bocaue, Bulacan 3018, \\ Philippines \\ ${ }^{2}$ Research Director, Basic Education Department, Dr. Yanga's Colleges Inc., Wakas, Bocaue, \\ Bulacan 3018, Philippines
}

\begin{abstract}
In 2015, Transport Network Companies (TNC) such as Grab and Uber were introduced in the Philippines. With the worsening traffic management system in the country, TNC units use the power of an application to cater the commuting needs and demands of the Filipino commuting public following a car-sharing principle. Taking into account their startup success, the conventional taxi industry has been struggling. On this basis, this phenomenological study therefore involves an in-depth understanding of the struggles and subsequent condition of the taxi industry amid operations of TNCs. With the use of snowball and convenience sampling, the study involved semi-structured interviews with drivers of conventional taxi, commuters, and the Philippines' Land Transportation Franchising and Regulatory Board (LTFRB) members to obtain perspectives from the regulating body. Employing Billups'(2016) qualitative data analysis method, six themes emerged including Effects of Transport Network Companies, Protests and Complaints of Taxi Drivers, Consequent Actions of Taxi Drivers due to Income Reduction, Motivations for Taxi Driving Business Continuity, Perceptions of Taxi Drivers on TNVS, and Commuter Perspective on both Transport Services. Based on the findings, it is concluded that since the TNCs introduction, the conventional taxi industry has been bombarded of varied issues relating to the quality of transport service they provide in terms of convenience, comfort and ease, affordability, and accessibility. These findings then translate to the need of a partial deregulation that involves amendments on some memorandum circulars, and the pioneered Code of Ethics for the entire Philippine Taxi Industry so as to sustain and correspondingly compete with TNCs in relation to the demands of the market.
\end{abstract}

\section{Introduction}

* Corresponding author: neiljohnbeltran.325@gmail.com 
Uber and Grab are popular modes of car sharing for commuters which were originally conceptualized in Malaysia and United States respectively [1] which are becoming popular in the commuting public of Metro Manila. The services that they provide is classified as Transport Network Companies (TNCs) while their partners are called Transportation Network Vehicle Services (TNVS). TNCs started operating in Manila streets in 2015 via the accreditation of the Land Transportation Franchising and Regulatory Board (LTFRB).

When TNCs entered the country, commuters noticed the difference right away. They changed service standards and pricing expectations and execution, using technology to resolve recurring problems with public transportation. As a matter of fact, findings from a study by Paronda et al. (2017) revealed that $83 \%$ of daily commuters said they would be willing to take TNVS recognizing its efficiency and convenience, while $17 \%$ answered they prefer a conventional taxi. In a separate study conducted by Paronda et al., it was estimated that 9,735 TNVS are moving into the Manila streets in contrast to 3,628 LTRFB-accredited conventional taxis with non-accredited cabs excluded. TNCs indeed have gained tremendous following due to the convenience, safety, and accessibility of the services they provide. With the recent issue on them being addition to the increasing volume of vehicles, they have now ventured into pooling rides to achieve better load efficiency and reducing costs for commuters going to similar destinations. These developments have even encouraged some private car owners to leave cars at home, a welcome development in reducing traffic. However, the rise of these car-sharing vehicles has been thought to have been grabbing the market once controlled by conventional taxis. The TNCs upended the transportation equation and disrupted the conventional taxi industry.

A recent development in March 2018 occurred when Grab wiped out Uber in the entire Southeast Asia by purchasing the latter. The combination of the two brings both beneficial and detrimental implications to the taxi industry according to Phillip Capital Research (Tang, 2018). Although, a lot believe that it will be more of an uplift for the disrupted taxi industry, since fare prices are foreseen to go up as Grab and Uber just integrated.

With these opposing aspects of the situation coupled with all the pre-data gathered information, this research therefore intends to identify and understand the present struggles and condition of the taxi industry taking into account the enforcement of Transport Network Companies in the country. Given the policy-related battles of both transportation services, the researchers probed more on the knot to understand better the perspectives of drivers, commuters, and of the government regarding the matter.

\section{Research methods}

Guided by its primordial objective, this study utilized a descriptive phenomenological form of inquiry. Such design allowed the researchers to explore experiences and sensory perception of the researched phenomenon and delve into perceptions, perspectives, understandings, and feelings of those people who have actually experienced or lived the phenomenon or situation of interest. Operating a purposive and convenience sampling, nine drivers of conventional taxi who have been driving such for at least 5 years were the primary and eligible informants of this study. The recruited informants were from varied and major areas in Metro Manila including Tondo, Sta. Mesa, and Quezon City. They were interviewed one at a time using a validated semi-structured instrument focused on their experiences as conventional taxi drivers. Data were then transcribed and scrutinized to search for meanings and pattern for data analysis. The researchers utilized Billups' [2] model of qualitative data analysis. Initially, the researchers organized the data through transcription. After so, annotation was done in order to construct the possible formulated meanings of the study. This was followed by identifying themes which were all summarized. Consequently, with some of the important data already in mind, these themes 
were integrated and findings were systematically shown following their specific theme. This assignment of themes were done through utilizing mutually-exclusive categories. The comprehensive analysis of data followed where clarifications and further data treatment was applied for the understanding of the readers. Lastly, all organized and thematically arranged data were interpreted.

A number of strategies were also employed to add rigor to the study such as "member checking" which was achieved by getting agreement from the informants on the emerged findings. In addition, the researcher reflected their own presuppositions about the perceptions of conventional taxi drivers using bracketing. Data triangulation was also employed by involving taxi operators and LTFRB personnel as sources of information. This was furthered by including commuters as a data source so as to gain an understanding on how the emergence of TNCs influence their perceptions about taking a taxi.

\section{Findings and discussions}

After a rigorous and comprehensive analysis of the data obtained, six emergent themes were developed that reflect the struggles and subsequent condition of the Metro Manila taxi industry amidst the current operations of Transport Network Vehicle Services. The first five themes present all the experiences of taxi drivers, while the sixth theme manifests all the data gathered from commuters.

\subsection{Effects of transport network companies to the Taxi Industry}

Table 1. Effects of Transport Network Companies.

\begin{tabular}{|l|l|}
\hline \multicolumn{1}{|c|}{ Theme Cluster } & \multicolumn{1}{c|}{ Formulated Meanings } \\
\hline \multirow{2}{*}{ Major Effect on Philippine Transportation } & Worsening Traffic Congestion \\
\cline { 2 - 2 } Fundamental Aftermath on Taxi Drivers & Illegally Operating Vehicle Prevalence \\
\hline \multirow{2}{*}{$\begin{array}{l}\text { Additional Effect in Daily Transaction of } \\
\text { Taxi Drivers }\end{array}$} & Decreasing Daily Income \\
\cline { 2 - 2 } & Contracted Transaction Prevalence \\
\cline { 2 - 2 } Driving Business Transpose & Inconsistency in Daily Operation \\
\hline \multirow{2}{*}{ Serving Transaction } \\
\cline { 2 - 2 } & Taxi Drivers Family Operating in TNCs \\
\cline { 2 - 2 } & Taxi Drivers Shifting to TNCs \\
\cline { 2 - 2 } & Acquaintance and Personal Urge to Shift to TNCs \\
\hline
\end{tabular}

This emergent theme tackles the general effects of TNCs to the entire taxi industry. Contrary to the notion that TNCs help resolve traffic problem in Metro Manila, TNCs contributed to the problem as highlighted in the findings. This is in relation to the idea that their presence added volume of vehicles in a very congested Metro Manila. The same was also cited by Schaller [3] who conducted an analysis about the growth of app-based vehicles in New York City. Her results disclosed that the total combined mileage of TNVS, yellow cabs, black cars, and car services increased from 14 to 19 percent inclusive of years 2013 to 2016. This then relates to the concept that they may have worsened the traffic congestion in Metro Manila. Furthermore, in Manhattan, TNCs have added an approximate $7 \%$ in the current miles consumed by all vehicles. Such numbers emphasize that these TNVS have direct and indirect impact to transportation as also evident in Avendano's (2017) work which emphasized the TNCs aggravation to traffic jam. Moreover, in 2017, travel time in Metro Manila was seen to become 5\% faster since Uber was suspended which merely points out that with those TNCs in the road, the traffic became more jammed. With 
this, the informants elaborated that the addition of TNVS to the road has made the traffic congestion in Manila worse as displayed in the next statement:

"It has a huge impact since it congested more the traffic flow. Even with the widening of the road, the traffic does not get better due to the number of vehicles. Most especially during rush hour. Sometimes there is a complete standstill and this can be attributed to the presence of Grab and Uber units now."

Another theme cluster relates to the financial effects of the presence of TNVS to the taxi drivers and operators. Collectively, the informants expressed concerns on the economic consequence with TNVS sharing their market. Similar to this concept is seen in Chang's [4] work whose study results showed that the regular revenue of taxi drivers is reduced by estimated $12 \%$ in the first year of Uber operation and $18 \%$ in its third year. Likewise, findings of Jiang and Zhang (2012) revealed that the taxi industry was greatly affected in terms of passenger accommodation average number which dropped by $18.08 \%$ and the income per driver depleted by $19.29 \%$ in the year 2015 . In the same way, the taxi drivers in Metro Manila are faced with income reduction that also resulted to debt infliction. This effect to taxi drivers is also confirmed by the informants as indicated in the next statement:

"It (income) has significantly decreased. Before, the income in taxi driving was good. But, with their (TNVS) presence, you are fortunate to earn Php1000 for a 24 hours of taxi driving."

In addition, another major effect of the presence of TNVS is taxi drivers relying on contractualized transaction and services to commuters which violate existing operating guidelines and policy concerning the taxi industry (Salvador, 2018). According to the Philippine Taxi Passenger Bill of Rights, taxi drivers are not entitled to have multiple hiring and are compelled to give official receipts in order to preserve the integrity of the drivers. Because of this, taxi drivers are forbidden to have such transactions since not all transactions can be recorded. This scenario has worsened when TNVS were introduced in the country. Since TNVS resulted to income reduction among taxi drivers, they tend to rely heavily on servicing and contractualized transaction to meet their target boundary fee (a fixed amount paid by drivers to the taxi company for driving the passenger vehicle) despite knowing that this is a violation. This formulated meaning is gathered through the next statement:

"Most taxi drivers do this, before agreeing to service a commuter, a contract will be made as to the cost of the transaction, if an amount is agreed, then they provide the service."

With all these playing in the field of taxi industry, some drivers have shifted and transferred or at least consider transferring to TNCs. There are confirmations about taxi drivers switching to Grab and Uber as an impact of TNCs to their operations. Similar effect was also observed in Chicago [5] where recent statistics showed that taxi drivers transferring to Uber in the year 2012 peaked to 538. Some of the taxi drivers in Manila have also shifted to TNCs as denoted through the following statement:

"Other taxi drivers are already operating as Uber and Grab drivers. They have ventured there because the profit has significantly reduced"

\subsection{Protests and Complaints of taxi drivers and operators}

Table 2. Protests and Complaints of Taxi Drivers and Operators.

\begin{tabular}{|c|l|}
\hline \multicolumn{1}{|c|}{ Theme Cluster } & \multicolumn{1}{c|}{ Formulated Meanings } \\
\hline \multirow{3}{*}{ Present Rules and Regulations of LTFRB } & Stringency in Guidelines \\
\cline { 2 - 2 } & Unfair Regulatory \\
\cline { 2 - 2 } & Unsystematic Regulation \\
\hline
\end{tabular}




\begin{tabular}{|l|l|}
\hline & Taxi Penalty System \\
\hline \multirow{4}{*}{ Taxi Drivers' Anticipated Actions of LTFRB } & No New Post-Policy Implementation \\
\cline { 2 - 2 } & Grounded Number of TNVS Units \\
\cline { 2 - 2 } & Performance Development \\
\cline { 2 - 2 } & No New Post-Policy Implementation \\
\hline \multirow{2}{*}{ Current Processing Steps of Both Industry } & Immediate TNVS Operation \\
\cline { 2 - 2 } & Different Processing Time Interval \\
\hline \multirow{2}{*}{ Irresolution and Indecision } & Negligence of Taxi-Reinforcing Groups \\
\cline { 2 - 2 } & No Representative in Government \\
\hline
\end{tabular}

The second emergent theme reflects all the supplications and complaints of taxi drivers in relation to the operations of Transport Network Companies. The theme elaborately identifies the struggles that the taxi drivers, operators, and the entire taxi industry.

The first theme cluster emphasizes the imbalanced and preferential treatment of LTFRB between TNCs and the taxi industry. This manifested when the agency temporarily suspended the admission and processing of TNVS application. Despite this, TNCs continued to accept drivers because of strong passenger demand thus operating without permits (Salvador, 2018). TNCs filed motions urging the agency, later agreed, not to apprehend unregistered TNVS until it decides on the appeal. This consequently resulted to taxi operators and drivers slamming what they called as LTFRB's preferential treatment saying that they should be spared too from apprehension while waiting for the approval of their franchise applications (Talabong, 2017). This stimulates the laments of taxi drivers.

The next theme cluster indicates all the viewpoints of taxi drivers regarding insufficiency of support in terms of policy amendments intended to solve their woes following the operations of TNCs. A case in point is the adjustment of flag down rate. In National Capital Region (NCR), the flagdown rate has been increased to Php40. Php13.50 is the charge per kilometer and Php2 will be added for the time travel every minute. In this case, it was presumed that these adjustments would greatly affect the effectiveness of services that the taxi industry provides. Still, many of the taxi drivers and operators perceive the whole industry is being neglected by the government. The industry demands a more sustainable support from the government to allow them to equally compete with TNVS. In the Philippines, there are still no new subsidies for the taxi drivers from the government since TNVS started. In the recent years, despite LTFRB's several extensive reviews of guidelines in TNCs, there are no new policies passed for taxi drivers magnifying the perception of the industry that they are neglected by the government (Carguide, 2018).

The next theme cluster on the other hand, reflects all the supplications of taxi drivers in the Philippines about the current processing steps for application of both taxi and TNCs. Presently, LTFRB issued an order allowing TNC drivers to operate even without permits like the Certificate of Public Convenience (Salvador, 2018). In addition, Inton (2017), a lawyer for Commuters Safety and Protection, mentioned that TNCs have a lot of applicants due to their facile process of admission. On the other hand, taxi applicants usually takes about 5 months before the franchise is processed. Hence, most of the taxi drivers complain towards the total difference between their processing time intervals. They believe that it is an unfair action of the authority.

\subsection{Consequent actions of taxi drivers for income reduction}

Table 3. Consequent Actions of Taxi Drivers for Income Reduction.

\begin{tabular}{|l|l|}
\hline \multicolumn{1}{|c|}{ Theme Cluster } & \multicolumn{1}{c|}{ Formulated Meanings } \\
\hline \multirow{2}{*}{ Strategic Action } & Double Time \\
\cline { 2 - 2 } & Overtime \\
\hline
\end{tabular}




\begin{tabular}{|l|l|}
\hline & Other Strategic Actions \\
\hline \multirow{2}{*}{ Taxi Operator and Driver Adjustments } & Meter Adjustments \\
\cline { 2 - 2 } & Target Boundary Amount Decline \\
\hline \multirow{3}{*}{ Other Alternative Source of Income } & Other Driving Income Source \\
\cline { 2 - 2 } & Sideline Business \\
\cline { 2 - 2 } & Second-Hand Taxi Trade \\
\hline \multirow{2}{*}{ Personal Angle } & Persistence \\
\cline { 2 - 2 } & Relationship Marketing \\
\hline
\end{tabular}

This theme specifically enumerates all the immediate actions of taxi drivers and operators to resolve the gradual decline of income brought forth by the success of TNVS in the country. It discusses the different strategies of taxi drivers and operators to address income depletion.

One identified strategy is taking advantage of the demands during peak hour of commuting. Taxi drivers claimed that they maximize their time and exploit opportunities during this specific period. A research by Yu [6] displayed that in China, there had been empirical data showing that taxi were seen to compete more effectively during peak ridesourcing periods which were 6 to $10 \mathrm{AM}$ and 5 to $8 \mathrm{PM}$ which usually happened to areas with high population density. This marks as one strategic action of cab drivers given the present dominance of app-hailing sedans. It enables taxi drivers to catch up with the daily target boundary amount which is eventually reduced by operators to aid in the economic adversity of the drivers.

Moreover, another important way to cope with the declining income, taxi drivers engage in sideline business. In the case of taxi drivers, they have also engaged in such in order to remain steadfast despite the surge of TNVS. Likewise, taxi drivers have employed other alternatives to generate more profit. One of those is driving other units after paying boundary fee for the prior unit. Additionally, some informants also narrated their desire to sell their units because of the economic effects of the emergence of TNVS. Informants said that this is an alternative way of resolving income loss.

On a more personal note, taxi drivers have practiced self-motivational strategies given the depletion of their daily income. Self-motivation is what drives a person directed towards realizing goals (Management Study Guide, n.d.). According to Marks (2014), taxi driving is a competitive business. It involves long hours of patience and hard work especially at times when they service impatient and unfriendly passengers. With this in mind, taxi drivers strongly believe that the combination of hard work and relationship marketing is needed in such situation.

\subsection{Motivations of taxi drivers for business continuity}

Table 4. Motivations of Taxi Drivers for Business Continuity.

\begin{tabular}{|l|l|}
\hline \multicolumn{1}{|c|}{ Theme Cluster } & \multicolumn{1}{c|}{ Formulated Meanings } \\
\hline \multirow{2}{*}{ Longstanding Business for Years } & Taxi Driving as Sole Source of Family Income \\
\cline { 2 - 2 } & Children's Education \\
\hline \multirow{4}{*}{ Anthropogenic Influence } & Relative Involvement \\
\cline { 2 - 2 } & Neighborhood Influence \\
\cline { 2 - 2 } & Family Member Financial Assistance \\
\hline \multirow{2}{*}{ Intrinsic Factor } & Age Intervention \\
\cline { 2 - 2 } & Health Consideration \\
\hline
\end{tabular}

This particularly involves different reasons why taxi drivers endure the identified struggles and decide to continue driving taxi as their primary income source despite the upsurge and public appreciation of Transport Network Vehicle service or TNVS. 
Amid the continuous popularity of TNVS in the Philippines, a lot of taxi drivers still choose to continue for multitude of reasons. One of the primary reasons is that taxi driving has been the sole source of income of their families for so long. In terms of family financial instability, their children's needs top the reasons why taxi drivers are willing endure the consequences of app-based vehicles. They have to stay in this industry since shifting to Transport Network Companies is costly. Instead of spending money for the shift, they would rather allot this to immediate family needs such as food and education. The taxi drivers rely on driving to financially provide for their respective families.

Another theme cluster that is formulated involves intrinsic factors regarding their business persistence. Other informants do not think much of shifting to TNCs or discontinuing their driving business since they have been doing this for so long. This gives them some sort of purpose and happiness especially taken into account their age where there are a lot of limitations. In an empirical study conducted by Jaen, Linan, and Moriano [7], it was eventually concluded that business owners are greatly influenced by their value priorities. Value priorities are principles that provide people with a way of knowing what is important to being happy. In this sense, the entrepreneurial values and intentions are said to be factors on why a business person is determined to keep going.

\subsection{Perceptions of Taxi Drivers about TNVS}

Table 5. Perceptions of Taxi Drivers about TNVS.

\begin{tabular}{|l|l|}
\hline Theme Cluster & \multicolumn{1}{c|}{ Formulated Meanings } \\
\hline \multirow{4}{*}{ TNVS as Favourable to Commuters } & Gives Greater Access to Transportation \\
\cline { 2 - 2 } & Gives Commuters' Conveniene \\
\cline { 2 - 2 } & Providing Easier Access to Commuters \\
\cline { 2 - 2 } & An Innovative Kind of Transportation Unit \\
\cline { 2 - 2 } TNCs as Another Service Providers & For Additional Transportation Means \\
\cline { 2 - 2 } in Philippine Transportation Industry & TNVS as Competitors \\
\cline { 2 - 2 } & No Competition \\
\hline
\end{tabular}

This emergent theme elaborates on how taxi drivers and operators alike perceive these appbased TNVS as another player in the field of public transportation service in the Philippines.

The theory proposed by Nobel Laureate Coase in 1991 [8] explains why commuters gradually transfer to TNCs. The theory connects to the idea of the application-based cars. TNVS make use of applications to accommodate riders. These applications are believed to be decreasing transaction cost as these increase the social utility of the passengers. This straightforwardly provides the reason why there is a continual public appreciation of TNCs since they provide greater availability using the application and decreases the transaction cost of commuters due to the fact that they use pre-arranged services. Likewise, taxi drivers also admit to all the advantageous features of TNVS that provide better services to commuters.

However, considering the context of the immediate popularity of TNCs, it may have disrupted the entire taxi industry. This is then considered a disruptive innovation, a concept proposed by Clayton M. Christensen. It is stated that a disruptive innovation is a powerful mean of developing new markets and providing new functionality that may disrupt an existing market (Godelnik, 2014). To most, these e-hailing applications disrupt the traditional taxi services. The Philippine National Taxi Operators Association even claimed that its members have lost around 20\% of their income (Mendoza, Ko, \& Mannipon, 2015). On another end, TNCs are perceived by the taxi drivers as an important competition. In the same way, competition is believed to be significant to all businesses (McCormick, 2016) since it encourages businesses to innovate and provide opportunities for improvement. 


\subsection{Commuter perspective on both transport services}

Table 6. Commuter Perspective on both Transport Services.

\begin{tabular}{|l|l|}
\hline \multicolumn{1}{|c|}{ Theme Cluster } & \multicolumn{1}{c|}{ Formulated Meanings } \\
\hline Bad Experience & Increased Bill \\
\hline \multirow{2}{*}{$\begin{array}{l}\text { Factors and Consideration for } \\
\text { a Good Public Utility Vehicle }\end{array}$} & Security (Awareness to Driver's Profile) \\
\cline { 2 - 2 } & Accessibility and Conveniene (Easier Access to Transport) \\
\cline { 2 - 2 }
\end{tabular}

This theme evidently presents the personal experiences and the aspects through the lens of the commuting public.

This theme cluster is marked by negative experiences of commuters in a taxi unit. According to Brown (2015), when the TNVS started, the taxi operators and drivers are up in arms. The Metro Manila taxi system became disorderly.

This current theme cluster also determines the expectations and considerations of commuters about what is a good and quality public vehicle service. In a study conducted by Cantwell [9], it was determined that commuter satisfaction is influenced by evasion of crowded locations and unreliable service. In addition, factor such as the length of the waiting time also affects commuter satisfaction. Moreover, of all the meanings that were gathered about the expectations of the commuters about a good public utility vehicle, security tops the list. Commuters believe on the importance of knowing the profile of the drivers. On a similar context, results of the study by Adriano and $\mathrm{Su}$ [10] revealed that security and convenience remain as primary considerations of passengers for choosing either street-hailed cabs or e-hailed cabs.

\section{Conclusions}

Taxi drivers along Metro Manila greatly perceive the beneficial importance of TNVS to the daily commuters since these mobile application-based transport system accommodates passengers with greater comfort, accessibility, and convenience. Security and integrity of drivers are two essential key points of consideration of commuters as well. However, they also recognize TNCs as a strong competition since they both offer transport services to the same market which greatly disrupted the entire taxi industry.

On the basis of all the findings, it can be inferred that the struggles and subsequent conditions of taxi drivers do not truly root from Transport Network Companies such as Grab and Uber. In fact, taxi drivers even recognized that TNCs are truly significant for passengers today. Living in the 21 st century mostly entails instant and convenient transactions due to the increasing demands and expectations of people. The advent of technology has greatly made the industry cope with the need of the commuting public. Thus, the industry too shall make adjustments and immerse itself in these innovations so as to provide better services to the public. LTFRB must implement reform in its regulatory processes for the taxi industry. This may include mobile application installment that can also be used by taxi drivers and commuters for better services. In addition, provision for a partial deregulation in Metro Manila taxi industry. As adjunct to that, the problem really roots from the outdated regulations of the government.

Relevantly, these findings have also led the researchers to the construction of the Codes of Ethics of Drivers. Clearly, there are no stated codes of ethics of taxi drivers in the Republic Act 4136 which only enumerates all the laws of land transportation and traffic rules. Thus, one of the recommendations emanating from this is to pioneer a version of the Codes of Ethics and Conduct of taxi drivers in the Philippines. This has also coincided with the findings in theme 6 about the experiences and preferences of commuters. 


\section{References}

1. Paronda A (2017). An Exploratory Study on Uber, GrabCar, and Conventional Taxis in Metro Manila. Conference: Sustainable Built Design 2017 ResearchGate pp 1-7 (Metro Manila)

2. Billups F D 2012 Qualitative Data Analysis: An Overview for Beginning Qualitative Researchers The NERA Researcher 50 pp 8-10

3. Schaller B (2017, February 27). Unsustainable? The Growth of App-Based Ride Services and Traffic, Travel and the Future of New York City. Retrieved (2018) from http://www.schallerconsult.com/rideservices/unsustainable.pdf

4. Chang H H 2017 The Economic Effects of Uber on Taxi Drivers in Taiwan. Journal of Competition Law and Economics 13 (Taiwan)

5. Wallsten S (2015) The Competitive Effects of the Sharing Economy: How is Uber Changing Taxis? Technology Policy Institute page 16 (New York; Washington, DC)

6. Yu M (2017, June) How can the taxi industry survive the tide of ridesourcing? Evidence from Shenzhen, China Transportation Research: Emerging Technologies 79 page 1 (Shenzhen, China)

7. Jaen, Liñan, \& Moriano 2010. Personal Values and Entrepreneurial Intentions: An Empirical study. Researchgate pp 438-453

8. Jenk, J (2015). Theory meets practice in the taxi industry: Coase and Uber. MPRA. page 2.

9. Cantwell M, et al. 2009 Examining the Factors that Impact Public Transport Commuting Satisfaction The Journal Public Transportation 12 pp 1-21 (South Florida)

10. Adriano M M \& Su C C 2017 Out with the Old, In with The New: A Study on The Vehicle Hailing Preferences of Filipino Taxi Riders Based on Participation Intent International Journal of Real Estate Studies 11 page 80 (University of the Philippines, Manila) 